\title{
Optimization of Heteronuclear Relayed Coherence-Transfer Spectroscopy
}

\author{
SuSANTA K. SARKAR AND AD BAX \\ Laboratory of Chemical Physics, National Institute of Arthritis, Diabetes, and Digestive \\ and Kidney Diseases, National Institutes of Health, Bethesda, Maryland
}

Received January 22, 1985

\begin{abstract}
The effectiveness of the heteronuclear RELAY experiment is analyzed for a number of different spin systems. It is found that the sensitivity of the measurement strongly depends on the duration of the mixing period and on a proper choice for the sampling time in the $t_{1}$ dimension. Good agreement is found between theoretical evaluations and experimental results, obtained for samples of propanol and cyclosporin A. (C) 1985 Academic Press, Inc.
\end{abstract}

\section{INTRODUCTION}

The heteronuclear RELAY experiment, first introduced by Bolton and Bodenhausen $(1,2)$, can be considered a combination of the homonuclear COSY experiment (3-7) and the heteronuclear chemical-shift-correlation experiment (710). In this experiment transfer of coherence occurs in two steps. For a typical ${ }^{1} \mathrm{H}-$ ${ }^{1} \mathrm{H}-{ }^{13} \mathrm{C}$ RELAY experiment the magnetization is first transferred from a distant proton to a proton that is directly attached to a ${ }^{13} \mathrm{C}$ nucleus. This transferred magnetization is then relayed from the attached proton to the ${ }^{13} \mathrm{C}$ nucleus. This method allows adjacent protonated ${ }^{13} \mathrm{C}$ nuclei to be identified $(1,2,11)$, provided that COSY type transfer between the two protons is feasible, i.e., provided that the vicinal ${ }^{1} \mathrm{H}-{ }^{1} \mathrm{H}$ coupling is well resolved.

The RELAY experiment is useful in cases where the resonances in the ${ }^{1} \mathbf{H}$ spectrum are overlapped so severely that individual resonance assignment is not possible. The use of the RELAY experiment for the study of peptides has recently been demonstrated $(11,12)$. However, it is commonly found that in many applications of this technique, the sensitivity of the experiment is considerably lower than that of the heteronuclear shift correlation experiment (10). Also, intensities of relayed and nonrelayed signals are generally not well understood. In this paper a detailed analysis of the RELAY experiment will be presented and general guidelines for the optimization of the experiment for different spin systems will be discussed.

The operator formalism (13-15) will be used to analyze the behavior of the spin system in our discussion of the experiment. In agreement with conventions adapted by Sørensen et al. (13), a pulse is defincd to rotate magnetization counterclockwise, i.e., a $90_{x}^{\circ}$ pulse rotates magnetization from the positive $z$ to the negative $y$ axis. 


\section{THE HETERONUCLEAR RELAY EXPERIMENT}

A number of slightly differcnt versions of the heteronuclear RELAY experiment have been proposed in the literature $(1,2,11,16)$. In this paper the modified sequence proposed by Bax (16) and Kessler et al. (11) will be analyzed. The pulse sequence is sketched in Fig. 1 and its higher sensitivity is an advantage over the original experiment (1). The effect of this sequence will be analyzed for the simple case of a weakly coupled AMPX spin system, where A, M, and P are protons with $\mathrm{M}$ coupled to both $\mathrm{A}$ and $\mathrm{P}$ and $J_{\mathrm{AP}}=0$ (Fig. 2). Throughout this paper, $\mathrm{X}$ is the ${ }^{13} \mathrm{C}$ nucleus, directly coupled to proton, M. The assumption $J_{\mathrm{AX}}, J_{\mathrm{PX}} \ll J_{\mathrm{MX}}$ is made in the following discussions. Application to more complicated systems is simple once the experiment is analyzed for the AMPX case.

In the heteronuclear RELAY experiment, magnetization is transferred from distant proton, $A$, to attached proton, $M$, and this transferred magnetization is then relayed to its directly coupled ${ }^{13} \mathrm{C}$ nucleus, $\mathrm{X}$. The correlation between distant protons and ${ }^{13} \mathrm{C}$ nuclei is the information of interest in a RELAY spectrum. Therefore, for the AMPX system, the most valuable information is provided by the transfer of magnetization from $A$ to $X$ (and from $P$ to $X$ ).

The RELAY of magnetization at various stages of the pulse sequence (Fig. 1) is outlined below. The first $90^{\circ}{ }^{1} \mathrm{H}$ pulse rotates the A spin magnetization to the $-y$ axis (time a):

$$
I_{\mathrm{A} z} \stackrel{90^{\circ}}{\rightarrow}-I_{\mathrm{A} y}
$$

This transverse magnetization evolves during the evolution period under influence of the Hamiltonian, $\Omega_{\mathrm{A}} I_{\mathrm{A} z}+2 \pi J_{\mathrm{AM}} I_{\mathrm{Az}} I_{\mathrm{Mz}}$, and at the end of the evolution period (time $\mathrm{b}$ ) the $\mathrm{A}$ spin magnetization is described by

$$
\begin{aligned}
-I_{\mathrm{A} y} \stackrel{\Omega_{\mathrm{A}} I_{\mathrm{A} z} t_{1}+2 \pi J_{\mathrm{AM}} I_{\mathrm{A} z} I_{\mathrm{M} z} t_{1}}{\longrightarrow}\left[I_{\mathrm{A} x} \sin \left(\Omega_{\mathrm{A}} t_{1}\right)-I_{\mathrm{A} y} \cos \left(\Omega_{\mathrm{A}} t_{1}\right)\right] \cos \left(\pi J_{\mathrm{AM}} t_{1}\right) \\
+2\left[I_{\mathrm{A} x} \cos \left(\Omega_{\mathrm{A}} t_{1}\right)+I_{\mathrm{A} y} \sin \left(\Omega_{\mathrm{A}} t_{1}\right)\right] I_{\mathrm{Mz} z} \sin \left(\pi J_{\mathrm{AM}} t_{1}\right) .
\end{aligned}
$$

The $180^{\circ}{ }^{13} \mathrm{C}$ pulse at the midpoint of the evolution period refocuses the effect of heteronuclear coupling and therefore, coupling between spin $\mathrm{A}$ and $\mathrm{X}$ is not included in the Hamiltonian that is used in expression [2]. The first term at the

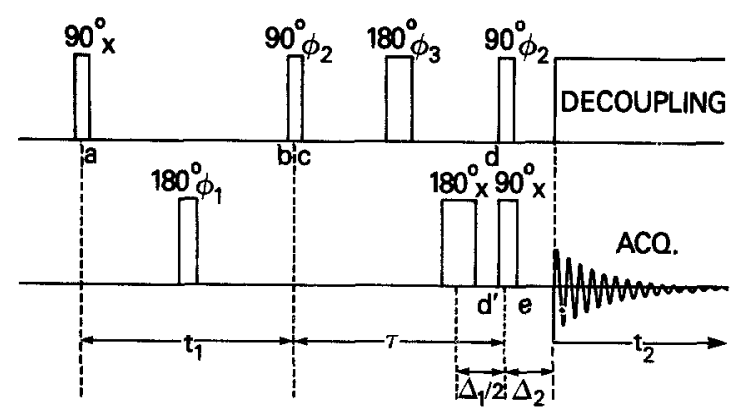

FiG. 1. Pulse scheme of the heteronuclear RELAY experiment. The phases of the if pulses and receiver are cycled according to Table 1 . 


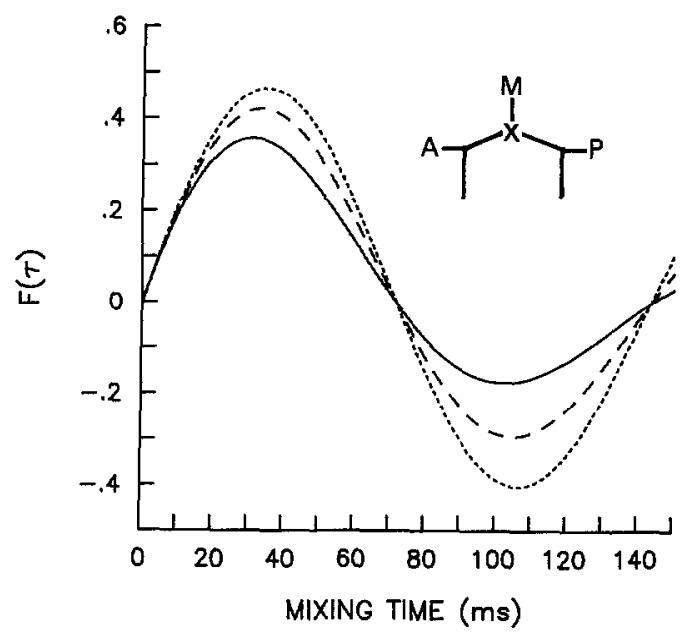

FIG. 2. Dependence of the transfer function, $f(\tau)$, on the duration of the mixing period, $\tau$, for an AMPX spin system. The function is sketched for values of $100 \mathrm{~ms}$ (solid line), $200 \mathrm{~ms}$ (broken line), and $500 \mathrm{~ms}$ (dotted line) for the transverse relaxation time, $T_{2 \mathrm{M}}$, of proton M. A function value, $f(\tau)=1$, corresponds to the case where all magnetization that is transferred from $A$ to $M$, is also relayed to carbon $\mathrm{X}$. Both delays, $\Delta_{1}$ and $\Delta_{2}$, are assumed to be equal to $1 /\left(2 J_{\mathrm{MX}}\right)$.

right-hand side represents the vector sum of the A spin-doublet components. The second term represents the antiphase part of the two doublet magnetization vectors which can, in part, be transferred to spin $\mathrm{M}$ by means of the second $90^{\circ}{ }^{1} \mathrm{H}$ pulse (time c):

$$
\begin{aligned}
2\left[I_{\mathrm{A} x} \cos \left(\Omega_{\mathrm{A}} t_{1}\right)+\right. & \left.I_{\mathrm{A} y} \sin \left(\Omega_{\mathrm{A}} t_{1}\right)\right] I_{\mathrm{M} z} \sin \left(\pi J_{\mathrm{AM}} t_{1}\right) \stackrel{90^{\circ}}{\rightarrow} \\
& -2 I_{\mathrm{A} x} I_{\mathrm{M} y} \cos \left(\Omega_{\mathrm{A}} t_{1}\right) \sin \left(\pi J_{\mathrm{AM}} t_{1}\right)-2 I_{\mathrm{A} z} I_{\mathrm{M} y} \sin \left(\Omega_{\mathrm{A}} t_{1}\right) \sin \left(\pi J_{\mathrm{AM}} t_{1}\right)
\end{aligned}
$$

The first term at the right-hand side of [3] denotes two-spin (zero- and doublequantum) coherence and cannot contribute to the transfer of magnetization from A to $\mathrm{X}(17)$. The second term, $I_{\mathrm{A} z} I_{\mathrm{M} y}$, represents the $\mathrm{M}$ spin magnetization transferred from $A$ that is in antiphase along the $\pm y$ axis. Assuming first, for reasons of simplicity, that no ${ }^{13} \mathrm{C} 180^{\circ}$ pulse is applied during the mixing period, $\tau$, the $180^{\circ}{ }^{1} \mathrm{H}$ pulse applied at the midpoint of this interval removes the effects of ${ }^{1} \mathrm{H}$ chemical shift and heteronuclear coupling during this period. However, evolution due to homonuclear coupling is not affected by this pulse. Since $M$ is coupled to both $\mathrm{A}$ and $\mathrm{P}$, the transverse $\mathrm{M}$ spin magnetization will evolve under the influence of the Hamiltonian, $2 \pi J_{\mathrm{AM}} I_{\Lambda z} I_{\mathrm{Mz}}+2 \pi J_{\mathrm{MP}} I_{\mathrm{M} z} I_{\mathrm{Pz}}$. Just before the $90^{\circ}{ }^{1} \mathrm{H},{ }^{13} \mathrm{C}$ pulse pair (time d) one obtains:

$$
\begin{aligned}
& 2 I_{\mathrm{M} y} I_{\mathrm{A} z} \stackrel{2 \pi J_{\mathrm{AM}} I_{\mathrm{M} z} I_{\mathrm{A} z} \tau+2 \pi J_{\mathrm{MP}} I_{\mathrm{M} z} I_{\mathrm{P} z} \tau}{\longrightarrow}\left[-I_{\mathrm{M} x} \sin \left(\pi J_{\mathrm{AM}} \tau\right) \cos \left(\pi J_{\mathrm{MP} \tau)}\right.\right. \\
& -2 I_{\mathrm{My} y} I_{\mathrm{P} z} \sin \left(\pi J_{\mathrm{MP}} \tau\right) \sin \left(\pi J_{\mathrm{AM}} \tau\right)+2 I_{\mathrm{A} z} I_{\mathrm{M} y} \cos \left(\pi J_{\mathrm{AM}} \tau\right) \cos \left(\pi J_{\mathrm{MP}} \tau\right) \\
& \left.-4 I_{\mathrm{A} z} I_{\mathrm{M} x} I_{\mathrm{P} z} \cos \left(\pi J_{\mathrm{AM}} \tau\right) \sin \left(\pi J_{\mathrm{MP}} \tau\right)\right]
\end{aligned}
$$


where the coefficients, present in expression [3] have been omitted to simplify the expression. The $180^{\circ}{ }^{13} \mathrm{C}$ pulse, applied at a time $\Delta_{1} / 2$ before the two $90^{\circ}\left({ }^{1} \mathrm{H},{ }^{13} \mathrm{C}\right)$ pulses, flips the spin state of the ${ }^{13} \mathrm{C}$ nucleus. This makes it appear as if no heteronuclear coupling is present during the last interval, $\Delta_{1}$, of the mixing period. Thus, the $180^{\circ}{ }^{1} \mathrm{H}$ pulse will not completely refocus the effect due to $J_{\mathrm{MX}}$. For $\Delta_{1}=\left(4 J_{\mathrm{MX}}\right)^{-1}$, the in-phase $\mathrm{M}$ spin magnetization, present along the $x$ axis, is converted into antiphase magnetization (time $\mathrm{d}^{\prime}$ ) according to

$$
I_{\mathrm{M} x} \stackrel{-\pi I \mathrm{X} z I \mathrm{M} z}{\longrightarrow}-I_{\mathrm{M} y} I_{\mathrm{X} z} \text {. }
$$

The term at the right-hand side of expression [5] is completely transferred into transverse antiphase $\mathrm{X}$ spin magnetization by the simultaneous application of a $90_{x}^{\circ}\left({ }^{1} \mathrm{H}\right), 90_{x}^{\circ}\left({ }^{13} \mathrm{C}\right)$ pulse pair (time e):

$$
I_{\mathrm{M} y} I_{\mathrm{Xz}} \stackrel{90 \times\left({ }^{\circ} \mathrm{H}\right) ; 900_{x}^{\circ}\left({ }^{13} \mathrm{C}\right)}{\longrightarrow} I_{\mathrm{Mz}} I_{\mathrm{X} y}
$$

As is the case in heteronuclear shift-correlation spectroscopy $(8)$, proton decoupling cannot be started immediately after the ${ }^{13} \mathrm{C}$ detection pulse because of the antiphase nature of the two $\mathrm{M}$ spin doublet components. A delay, $\Delta_{2}$, equal to $1 /\left(2 J_{\mathrm{MX}}\right)$ for methine carbons, and $1 /\left(4 J_{\mathrm{MX}}\right)$ for methylene and methyl carbons is inserted before ${ }^{1} \mathrm{H}$ decoupling is started. This ensures that ${ }^{1} \mathrm{H}$-decoupled ${ }^{13} \mathrm{C}$ signals can be recorded.

The complete expression for the relayed signal with quadrature detection during $t_{2}$ is given by

$$
\begin{aligned}
S_{\mathrm{AX}}\left(t_{1}, t_{2}\right)=\sin \left(\pi J_{\mathrm{AM}} \tau\right) \cos \left(\pi J_{\mathrm{MP}} \tau\right) \sin \left(\pi J_{\mathrm{MX}} \Delta_{1}\right) \sin \left(\pi J_{\mathrm{MX}} \Delta_{2}\right) \\
\times \sin \left(\pi J_{\mathrm{AM}} t_{1}\right) \sin \left(\Omega_{\mathrm{A}} t_{1}\right) \exp \left(i \Omega_{\mathrm{X}} \Delta_{2}\right) \exp \left(i \Omega_{\mathrm{X}} t_{2}\right) .
\end{aligned}
$$

Analogous to phase cycling in the COSY (5-7) and heteronuclear shift-correlation experiments (9), incrementing the phases of all pulses in the mixing period by $90^{\circ}$, gives a signal of the form

$$
\begin{aligned}
S_{\mathrm{AX}}^{\prime}\left(t_{1}, t_{2}\right)=i \sin \left(\pi J_{\mathrm{AM}} \tau\right) \cos \left(\pi J_{\mathrm{MP}} \tau\right) & \sin \left(\pi J_{\mathrm{MX}} \Delta_{1}\right) \sin \left(\pi J_{\mathrm{MX}} \Delta_{2}\right) \\
& \times \sin \left(\pi J_{\mathrm{AM}} t_{1}\right) \cos \left(\Omega_{\mathrm{A}} t_{1}\right) \exp \left(i \Omega_{\mathrm{X}} \Delta_{2}\right) \exp \left(i \Omega_{\mathrm{X}} t_{2}\right) .
\end{aligned}
$$

Taking the difference of Eqs. [7] and [8] gives a signal modulated in phase as a function of $t_{1}$;

$$
\begin{aligned}
S_{\mathrm{AX}}^{-}\left(t_{1}, t_{2}\right)=\sin \left(\pi J_{\mathrm{AM}} \tau\right) \cos \left(\pi J_{\mathrm{MP}} \tau\right) & \sin \left(\pi J_{\mathrm{MX}} \Delta_{1}\right) \sin \left(\pi J_{\mathrm{MX}} \Delta_{2}\right) \\
& \times \sin \left(\pi J_{\mathrm{AM}} t_{1}\right) \exp \left(i \Omega_{\mathrm{A}} t_{1}\right) \exp \left(i \Omega_{\mathrm{X}} \Delta_{2}\right) \exp \left(i \Omega_{\mathrm{X}} t_{2}\right) .
\end{aligned}
$$

A four-step phase cycle can be used for additional suppression of axial peaks $(1,11$, 16). The phases of the ${ }^{1} \mathrm{H}$ and ${ }^{13} \mathrm{C} 180^{\circ}$ pulses were also cycled in our experiment to remove effects due to its spurious $90^{\circ}$ character (18), which can cause significant distortions in the observed RELAY intensities. Use of a composite $180^{\circ}{ }^{1} \mathrm{H}$ pulse (19-21) was not adequate on our instrument. The actual phase cycling used is presented in Table 1. 


\section{OPTIMIZATION OF EXPERIMENTAL PARAMETERS}

The factors to be considered for optimization of the heteronuclear RELAY experiment are (a) maximizing the intensity of the relayed signals, (b) minimizing the intensity of the nonrelayed signals, and (c) optimizing the resolution in the $2 \mathrm{D}$ spectrum. These points will be addressed below.

\section{Maximizing RELAY Intensity}

The amount of magnetization relayed from proton, $\mathrm{A}$, to carbon, $\mathrm{X}$, depends on how much magnetization is transferred from $A$ to $M$ and on the amount of magnetization that is transferred from $M$ to $X$. From expression [9], it is seen that for the AMPX system the dependence on the duration of the mixing period can be described by a transfer function, $f(\tau)$, given by

$$
f(\tau)=\sin \left(\pi J_{\mathrm{AM}} \tau\right) \cos \left(\pi J_{\mathrm{MP}} \tau\right) \exp \left(-\tau / T_{2 \mathrm{M}}\right)
$$

where $T_{2 \mathrm{M}}$ is the transverse relaxation time of spin, $\mathrm{M}$. The shape of this function is sketched in Fig. 2 for different values of the relaxation time, $T_{2 \mathrm{M}}$. A general expression for the transfer function (11) for an arbitrary spin system, $\mathrm{A}_{n} \mathrm{M}_{j} \mathrm{X} k$, where $\mathrm{M}$ is coupled to $\mathrm{A}$ and $\mathrm{X}$ and to a number of other spins, labeled $k$, is given by

$$
\begin{aligned}
f(\tau)=\prod_{k} \cos \left(\pi J_{\mathrm{M} k} \tau\right) \sin \left(\pi J_{\mathrm{AM}} \tau\right) \cos ^{n-1}\left(\pi J_{\mathrm{AM}} \tau\right) \sin \left(\pi J_{\mathrm{MX}} \Delta_{1}\right) \\
\times \cos ^{j-1}\left(\pi J_{\mathrm{MX}} \Delta_{2}\right) \sin \left(\pi J_{\mathrm{MX}} \Delta_{2}\right) \exp \left(-\tau / T_{2 \mathrm{M}}\right)
\end{aligned}
$$

TABLE 1

The Phases of the rf Pulses and of the Receiver in the Various Steps of the Heteronuclear RELAY Experiment ${ }^{a}$

\begin{tabular}{rrrrr}
\hline Step & $\phi_{1}$ & $\phi_{2}$ & $\phi_{3}$ & Receiver \\
\hline 1 & $x$ & $x$ & $x$ & $x$ \\
2 & $y$ & $x$ & $y$ & $-x$ \\
3 & $-x$ & $x$ & $-x$ & $x$ \\
4 & $-y$ & $x$ & $-y$ & $-x$ \\
5 & $x$ & $y$ & $y$ & $y$ \\
6 & $y$ & $y$ & $-x$ & $-y$ \\
7 & $-x$ & $y$ & $-y$ & $y$ \\
8 & $-y$ & $y$ & $x$ & $-y$ \\
9 & $x$ & $-x$ & $-x$ & $-x$ \\
10 & $y$ & $-x$ & $-y$ & $x$ \\
11 & $-x$ & $-x$ & $x$ & $-x$ \\
12 & $-y$ & $-x$ & $y$ & $x$ \\
13 & $x$ & $-y$ & $-y$ & $-y$ \\
14 & $y$ & $-y$ & $x$ & $y$ \\
15 & $-x$ & $-y$ & $y$ & $-y$ \\
16 & $-y$ & $-y$ & $-x$ & $y$ \\
\hline
\end{tabular}

${ }^{a}$ The pulse sequence is drawn in Fig. 1. 
In this expression, $n$ and $j$ denote the degeneracies of spins $\mathrm{A}$ and $\mathrm{M}$, respectively. For example, for transfer from the methyl protons to the adjacent methylene carbon in propanol $\left(\mathrm{CH}_{3} \mathrm{CH}_{2} \mathrm{CH}_{2} \mathrm{OH}\right), n$ equals 3 and $j$ equals 2 . Spins, $k$, are the two protons directly attached to the carbon that is bonded to the hydroxyl group. For the case where carbon, $X$, is directly attached to two nonequivalent methylene protons, $M$ and $Q$, the transfer function is the sum of two contributions, transfer via $M$ and transfer via $Q$ :

$$
\begin{gathered}
f(\tau)=\prod_{k} \cos \left(\pi J_{\mathrm{M} k} \tau\right) \sin \left(\pi J_{\mathrm{AM}} \tau\right) \cos ^{n-1}\left(\pi J_{\mathrm{AM}} \tau\right) \cos \left(\pi J_{\mathrm{MQ}} \tau\right) \sin \left(\pi J_{\mathrm{MX}} \Delta_{1}\right) \sin \left(\pi J_{\mathrm{MX}} \Delta_{2}\right) \\
\times \cos \left(\pi J_{\mathrm{MQ}} \Delta_{2}\right) \exp \left(-\tau / T_{2 \mathrm{M}}\right)+\prod_{k} \cos \left(\pi J_{\mathrm{Q} k} \tau\right) \sin \left(\pi J_{\mathrm{AQ}} \tau\right) \cos ^{n-1}\left(\pi J_{\mathrm{AQ}} \tau\right) \\
\quad \times \cos \left(\pi J_{\mathrm{MQ}} \tau\right) \sin \left(\pi J_{\mathrm{QX}} \Delta_{1}\right) \sin \left(\pi J_{\mathrm{QX}} \Delta_{2}\right) \cos \left(\pi J_{\mathrm{MQ}} \Delta_{2}\right) \exp \left(-\tau / T_{2 \mathrm{Q}}\right) . \quad \text { [12 }
\end{gathered}
$$

Transfer functions of these types are sketched in Fig. 3 for a number of different spin systems. These diagrams serve only to give an indication for what mixing time to choose in order to optimize a certain type of RELAY transfer; in actual use of the RELAY experiment one has to estimate the size of the couplings involved and calculate the transfer efficiency on the basis of Eq. [11] or [12].

A second factor, of major importance for maximizing the intensity of RELAY peaks in $2 \mathrm{D}$ spectra, is the transfer efficiency of magnetization from $n$ equivalent $\mathrm{A}$ spins, $\mathrm{A}_{n}$, to spin $\mathrm{M}$. For a spin system where $n$ equivalent spins, $\mathrm{A}$, are coupled to $\mathrm{M}$ and to a number of other protons, $r$, this transfer efficiency, as a function of $t_{1}$, is given by

$$
g\left(t_{1}\right)=n \prod_{r} \cos \left(\pi J_{\mathrm{A} r} t_{1}\right) \sin \left(\pi J_{\mathrm{AM}} t_{1}\right) \exp \left(-t_{1} / T_{2 \mathrm{~A}}\right) .
$$

The shape of this function is shown in Fig. 4 for a number of different spin systems. Again, for calculating the transfer efficiency as a function of $t_{1}$, one has to estimate the size of the couplings involved and substitute these in Eq. [13]. It is seen from Eq. [13] that the transfer efficiency as a function of $t_{1}$ oscillates strongly. Of course, in the $2 \mathrm{D}$ experiment $t_{1}$ values are systematically stepped between 0 and $t_{1_{\max }}$ and it is the average of $g\left(t_{1}\right)^{2}$ over this period that determines the total amount of signal power that will be present in the $2 \mathrm{D}$ spectrum. If $t_{1_{\max }}$ is long compared to the reciprocal of all couplings, $J_{\mathrm{A} r}$, this signal power will be spread over $2^{r+1}$ multiplet components in the $F_{1}$ dimension, decreasing the maximum peak intensity by a factor $2^{r}$ relative to the case where spin $\mathrm{A}$ is only coupled to $\mathrm{M}$. Alternatively, $t_{1_{\max }}$ can be chosen short to avoid this distribution of intensities. However, because of the factor $\sin \left(\pi J_{\mathrm{AM}} t_{1}\right)$ in Eq. [13], the RELAY intensity will be distributed over a minimum of two multiplet components that have an antiphase relationship. In the heteronuclear chemical-shift-correlation experiment, the appearance of multiplet splittings in the $F_{1}$ dimension (and consequent loss in sensitivity) can be avoided by using a $t_{1_{\max }}<\left(2 J_{111 I_{\max }}\right)^{-1}(10)$. For the RELAY experiment such a short $t_{l_{\max }}$ can lead to severe sensitivity loss since mutual cancellation of antiphase components in the $F_{1}$ multiplet structure will occur. Evaluation of Eq. [13] gives an idea about which $t_{1_{\max }}$ to select, and even in this case, the transfer of magnetization from A to $M$ can be an inefficient process. 

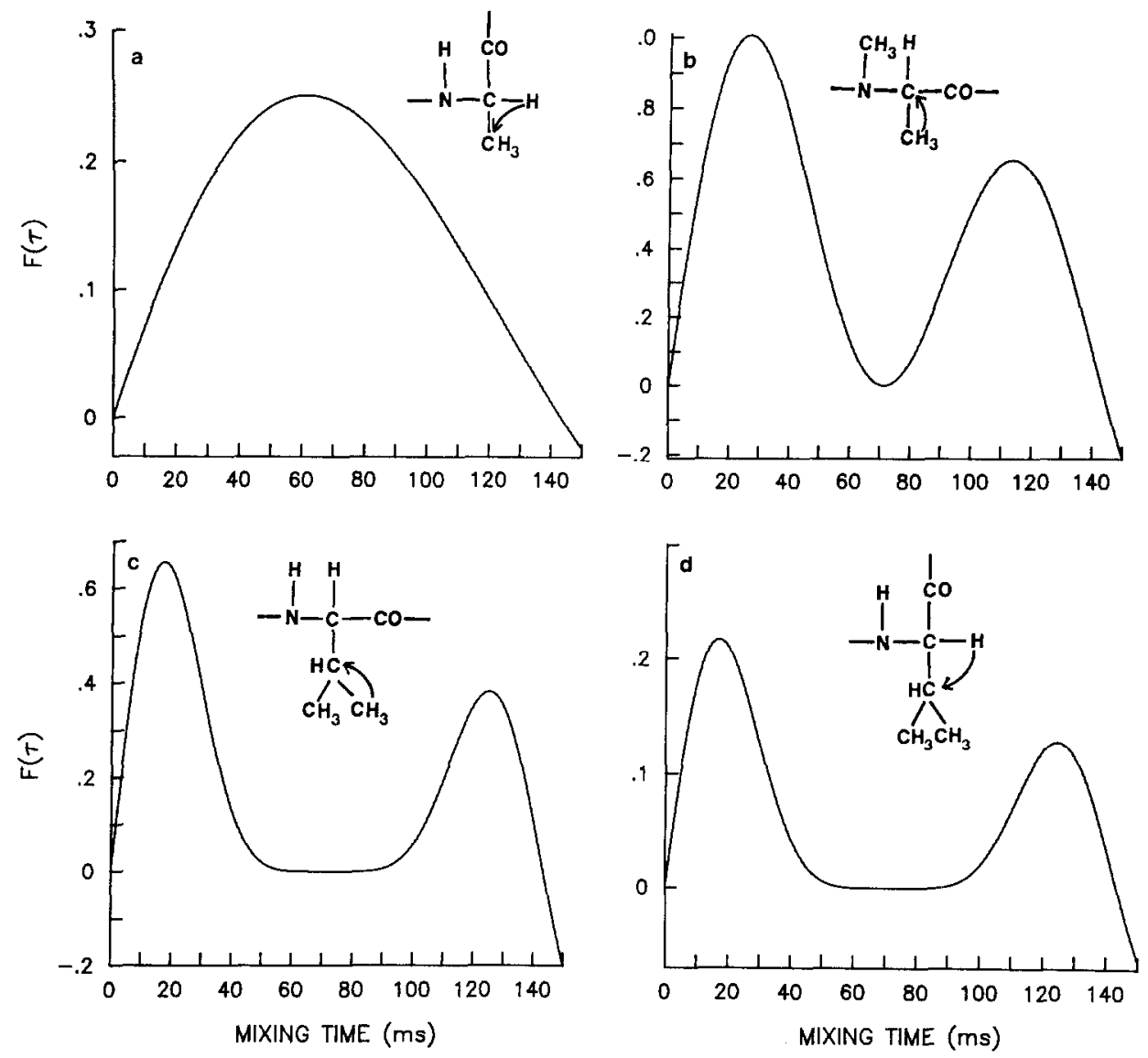

FIG. 3. Dependence of the transfer function, $f(\tau)$, on the duration of the mixing period for different spin systems, assuming a $T_{2}$ value for spin $\mathrm{M}$ of $200 \mathrm{~ms}$, and vicinal proton couplings of $7 \mathrm{~Hz}$. (a) $\mathrm{AM}_{3} \mathrm{X}$ system, for example, transfer of magnetization from the $\mathrm{C}_{\alpha}$ proton to the $\mathrm{C}_{\beta}$ methyl carbon in alanine. (b) $\mathrm{A}_{3} \mathrm{MX}$ system, for example, transfer from the $\mathrm{C}_{\beta}$ methyl protons to the $\mathrm{C}_{\alpha}$ carbon in $N$-methyl alanine. (c) $\mathrm{A}_{3} \mathrm{QMXP} \mathrm{P}_{3}$ system, for example, transfer from the $\mathrm{C}_{\gamma}$ methyl protons to the $\mathrm{C}_{\beta}$ carbon in valine. (d) $A M X P_{3} Q_{3}$ system, for example, transfer from the $C_{\alpha}$ proton to the $C_{\beta}$ carbon in valine. For all four curves, $\Delta_{1}$ and $\Delta_{2}$ values equal to $1 /\left(2 J_{\mathrm{MX}}\right)$ are assumed, with the exception of the $\mathrm{AM}_{3} \mathrm{X}$ system (curve (a)), where the calculation is based on a $\Delta_{2}$ value of $1 /\left(4 J_{\mathrm{MX}}\right)$.

\section{Minimization of Nonrelayed Signals}

The presence of intense resonances due to direct correlation, in our case the correlation between $\mathrm{M}$ and $\mathrm{X}$, can obscure the presence of weak RELAY peaks. A clever way to suppress such direct correlation peaks has been proposed by Kogler et al. (22). However, in practice, their modification can sometimes be inconvenient because of the large amount of phase cycling needed. If this modification is not used, partial suppression of direct correlations can be obtained by means of digital filtering. This will be briefly discussed below. The amount of $M$ spin magnetization that is generated by the first ${ }^{1} \mathrm{H}$ pulse (time a in Fig. 1) and is finally transferred to spin X, has a dependency, $h\left(t_{1}\right)$, on the duration of the evolution period, given by 

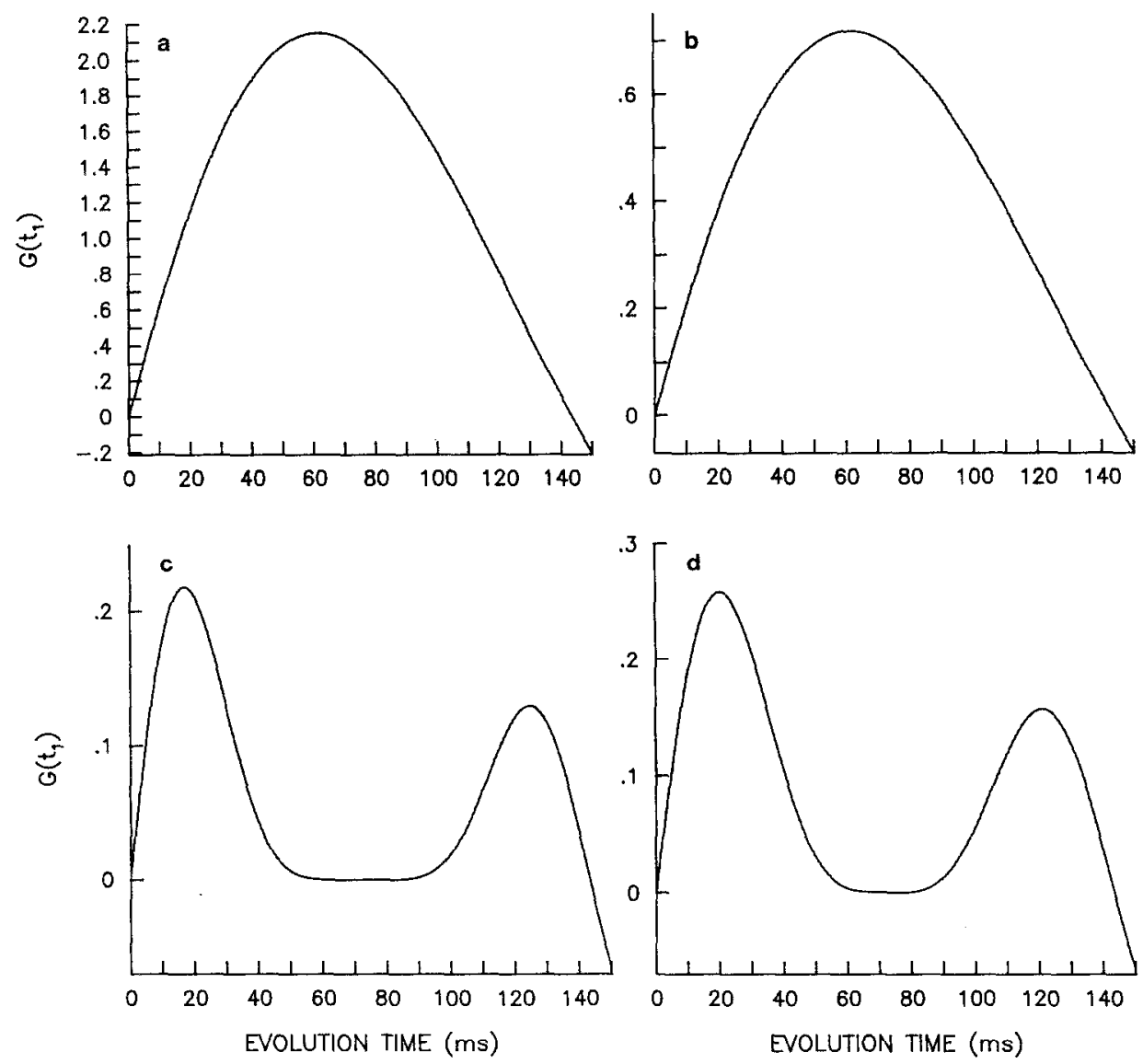

FIG. 4. The amount, $g\left(t_{1}\right)$, of magnetization that is transferred from proton(s) A, to proton(s) M, as a function of the evolution period, $t_{1}$ for different spin systems. The diagrams are based on $7 \mathrm{~Hz}$ vicinal proton couplings and a $T_{2 \mathrm{~A}}$ value of $200 \mathrm{~ms}$. Intensity " 1 " corresponds to the thermal equilibrium Boltzmann magnetization of a single proton. (a) $\mathrm{A}_{3} \mathrm{MX}$ system, for example, transfer from the $\mathrm{C}_{\beta}$ protons to the $\mathrm{C}_{\alpha}$ proton in alanine. (b) AMX system, for example, transfer of magnetization from the amide proton to the $C_{\alpha}$ proton in all amino acids (except glycine). (c) $P_{3} Q_{3} A M X$ system, for example, transfer from the $C_{\beta}$ proton to the $C_{\alpha}$ proton in valine. (d) $P_{3} Q A M_{3} X$ system, for example, transfer from the $C_{\beta}$ proton to the protons of one of the two $C_{\gamma}$ methyl groups in valine.

$$
h\left(t_{1}\right)=\prod_{k} \cos \left(\pi J_{\mathrm{M} k} t_{1}\right) \cos \left(\pi J_{\mathrm{AM}} t_{1}\right) \exp \left(-t_{1} / T_{2 \mathrm{M}}\right)
$$

where spins $k$ denote again all protons but $\mathrm{A}$, that are coupled to $\mathrm{M}$. Partial suppression of this type of signal can be obtained by using, in the $t_{1}$ dimension, a digital filter that has the shape of Eq. [13]. Such a digital filter provides matched filtering for the RELAY signal, whereas direct correlations are strongly attenuated. This type of filtering procedure has proven to be successful in the COSY experiment (23), and is also applicable in the heteronuclear RELAY experiment. Note however, that due to reasons explained in the previous section, the acquisition time in the $t_{1}$ 
dimension, $t_{1_{\max }}$, is often chosen rather short $(\sim 75 \mathrm{~ms})$, and consequently $g\left(t_{1}\right)$ will approximately have the shape of a sine bell (24), which is more convenient for practical use.

\section{Optimization of Resolution}

In the past, heteronuclear RELAY spectra have almost exclusively been recorded in the absolute value mode. This is convenient but not optimal as far as resolution and sensitivity are concerned. However, recording of pure absorption-mode spectra is impossible unless experimental modifications (25) are used that would cause a loss in sensitivity. In the 2D heteronuclear RELAY spectrum, peaks that correspond to the direct correlation between $M$ and $X$ and to the RELAY connectivity between $\mathrm{A}$ and $\mathrm{X}$ compare, respectively, with the diagonal and cross peaks in a COSY spectrum. These types of resonances are $90^{\circ}$ out of phase relative to one another, and additionally, the individual multiplet components in a COSY cross multiplet (which compare with AX RELAY) are in antiphase relative to one another. Presenting the RELAY spectrum in the absolute-value mode is therefore an acceptable alternative. To avoid tailing of the absolute-value-mode resonances, a sine-bell digital filtering window (24), or a pseudo echo window (26) can be used. As is cxplained in the previous section, such filtering functions have the additional advantage that they are close to matched filtering for the RELAY signals, whereas they strongly decrease the intensity of direct correlations.

\section{RESULTS AND DISCUSSION}

We have verified experimentally the agreement between the theoretical transfer functions and measured RELAY intensities. Experiments were performed on a $50 \%$ $\mathrm{v} / \mathrm{v}$ mixture of propanol in ${ }^{2} \mathrm{H}_{2} \mathrm{O}$ on a Nicolet $270 \mathrm{MHz}$ spectrometer, equipped with a $5 \mathrm{~mm}{ }^{13} \mathrm{C}$ probe. Nineteen two-dimensional experiments were performed with mixing times varying from 5 to $150 \mathrm{~ms}$. The intensities of the four observable RELAY peaks are depicted in Fig. 5, together with the theoretical curves that are based on Eq. [11]. Good agreement between theoretical and experimental values is found (Fig. 5) if complete phase cycling is used (Tablc 1). If not used, significant deviations from the theoretical curve were found, especially for relatively long mixing times.

The dependence of the AX RELAY peak intensity on the A spin multiplet structure is demonstrated for the valine and $N$-methyl valine residues in cyclosporin $A$, a compound investigated in detail by two-dimensional NMR methods (12). Using Eq. [11], magnetization RELAY has been optimized for transfer of magnetization from the $C_{\alpha}$ hydrogen to the $C_{\beta}$ carbon resonance, and a mixing time, $\tau$, of $16 \mathrm{~ms}$ is actually used. The $\mathrm{C}_{\alpha}$ proton of $N$-methyl valine is a doublet in the proton spectrum, with a coupling of $11.0 \mathrm{~Hz}$ to the $\mathrm{C}_{\beta}$ proton, whereas in valine the $\mathrm{C}_{\alpha}$ proton is a doublet of doublets with a coupling of $8.2 \mathrm{IIz}$ to the amide proton and $9.0 \mathrm{~Hz}$ to the $\mathrm{C}_{\beta}$ proton. On the basis of Eq. [13], it is evident that the RELAY intensity for the $N$-methyl valine should be significantly larger than for valine. This is experimentally confirmed. Figure 6 shows the region near the $\mathrm{C}_{\beta}{ }^{13} \mathrm{C}$ resonances 

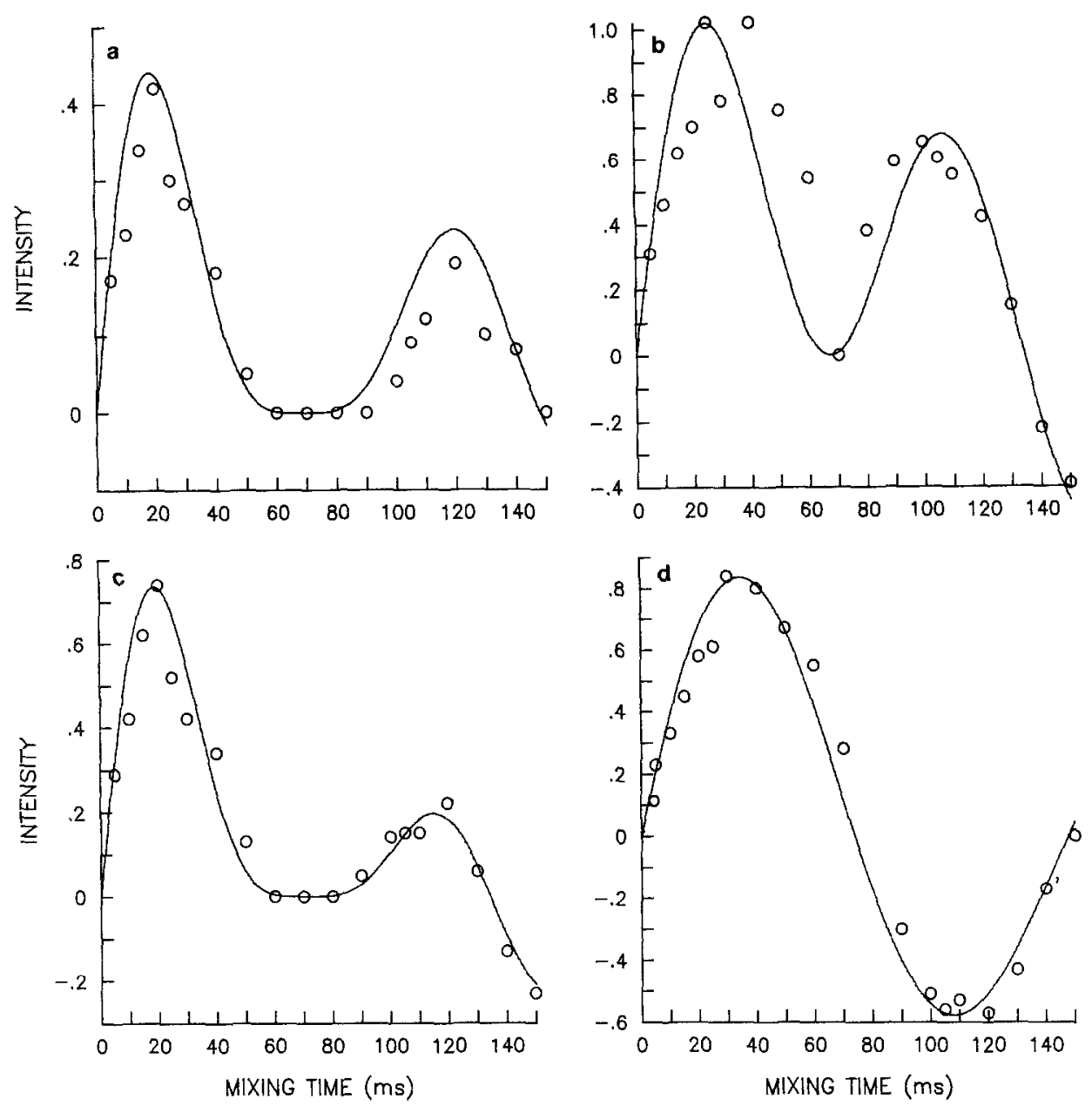

FIG. 5. Comparison of experimental and theoretical values of RELAY intensities as a function of mixing time, for a $50 \% \mathrm{v} / \mathrm{v}$ mixture of propanol $\mathrm{CH}_{3} \mathrm{CH}_{2} \mathrm{CH}_{2} \mathrm{OH}$. (a) Relay from the protons, $\mathrm{C}-1$, to the methylene carbon, C-2. (b) Relay from methylene protons, C-2, to methyl carbon, C-3. (c) Relay from methyl protons, C-3, to carbon C-2. (d) Relay from protons $\mathrm{C}-2$ to carbon $\mathrm{C}-1$. Open circles are the experimental points, and the solid lines represent the values based on Eq. [11]. All 2D spectra were recorded under identical conditions on a $270 \mathrm{MHz}$ spectrometer.

in the RELAY spectrum of a $150 \mathrm{~m} M$ solution of cyclosporin $\mathrm{A}$ in $\mathrm{CDCl}_{3}$, obtained at $500 \mathrm{MHz}$. An acquisition time of $85 \mathrm{~ms}$ has been used in the $t_{1}$ dimension, and a sine-bell digital filter was used. The two RELAY peaks of interest are marked by arrows, and clearly the valine RELAY peak has much lower intensity than the $N$ methyl valine RELAY peak. In the regular heteronuclear shift-correlation spectrum (not shown), the intensities of all correlation peaks between the $\mathrm{C}_{\alpha}$ protons and carbons are nearly identical for all amino acid residues if short acquisition times $(\sim 50 \mathrm{~ms})$ in the $t_{1}$ dimension are used. 


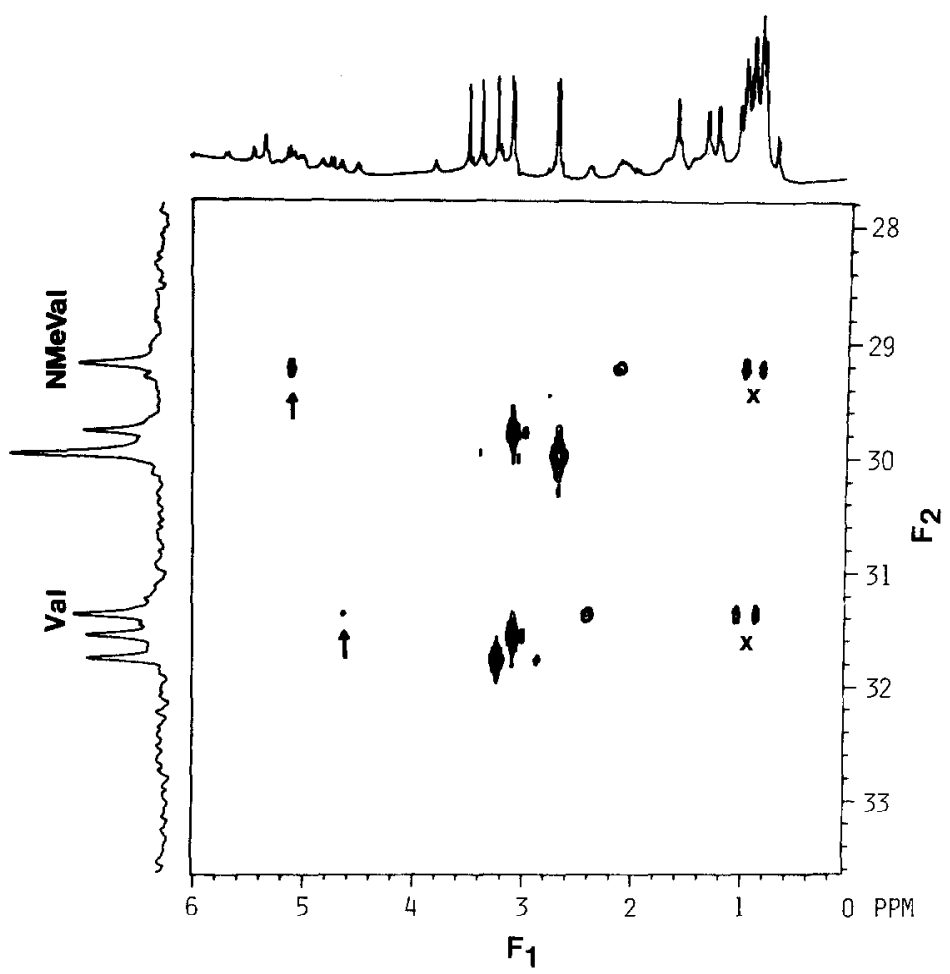

FIG. 6. Region of the $\mathrm{C}_{\rho}{ }^{13} \mathrm{C}$ resonances in the RELAY spectrum of cyclosporin $\mathrm{A}$ in $\mathrm{CDCl}_{3}$, recorded at $500 \mathrm{MHz}$. The RELAY peaks between the $\mathrm{C}_{\alpha}$ proton and the $\mathrm{C}_{\beta}$ carbon in the valine and $N$-methyl valine residues are indicated with arrows, and the RELAY peaks between the $C_{\gamma}$ methyl protons and the $\mathrm{C}_{\beta}$ carbon are marked " $\times . "$

Another interesting conclusion, drawn on the basis of Eqs. [11]-[13] and confirmed experimentally, is that it is generally much less sensitive to relay magnetization from a methine proton to an adjacent methylene or methyl carbon than from the methylene or methyl protons to the methine carbon. For example, RELAY peaks between the methyl protons and the $C_{\beta}$ carbon are very intense for both valine residues shown in Fig. 6, whereas sensitivity did not permit observation of RELAY peaks between the $C_{\beta}$ proton and the $C_{\gamma}$ methyl carbons, even when an optimum mixing time was used. Experimentally, we have found that the intensity of RELAY peaks is usually a factor of 3 to 10 lower than is the intensity of peaks in a heteronuclear shift-correlation spectrum, provided that both experiments are optimized for sensitivity, and recorded in identical measuring times. For saturated polycyclic hydrocarbons with many nonequivalent geminal protons, it is found on the basis of Eqs. [12]-[14] that it is very difficult to obtain effective magnetization RELAY. In unfortunate cases, magnetization RELAY can be as much as a factor of 20 lower than in the shift-correlation spectrum. This experiment nonetheless provides, even in this case, significantly better sensitivity than does the 2D INADEQUATE experiment (27-29). An alternative version of the heteronuclear 
RELAY experiment, named "pseudo double-quantum spectroscopy" (30), has the advantage that no choice for the duration of the mixing period has to be made, but provides pseudo-RELAY intensities that will be somewhat lower than obtained in the heteronuclear RELAY experiment under optimized conditions. This lower sensitivity is due to the fact that in the pseudo double-quantum experiment the mixing time is effectively varied between 0 and $t_{1_{\max }} / 2$, providing a less efficient mechanism for magnetization RELAY than does a single optimized duration.

\section{ACKNOWLEDGMENTS}

The authors are indebted to R. Tschudin for continuous technical support and thank Dr. H. Kessler for sending a preprint of Ref. (12).

\section{REFERENCES}

1. P. H. Bolton, J. Magn. Reson. 48, 336 (1982).

2. P. H. Bolton and G. Bodenhausen, Chem. Phys. Lett. 89, 139 (1982).

3. J. JEENER, Ampere International Summer School, Basko Polje, Yugoslavia, 1971.

4. W. P. Aue, E. Bartholdi, AND R. R. ERnst, J. Chem. Phys. 64, 2229 (1976).

5. K. Nagayama, A. Kumar, K. Wüthrich, and R. R. ERnSt, J. Magn. Reson. 40, 321 (1980).

6. A. BaX AND R. Freeman, J. Magn. Reson. 44, 542 (1981).

7. A. BAX, "Two-Dimensional Nuclear Magnetic Resonance in Liquids," Reidel, Boston, 1982.

8. A. A. MAudsley, L. MÜlLER, AND R. R. ERnst, J. Magn. Reson. 28, 463 (1977).

9. A. BAX AND G. A. Morris, J. Magn. Reson. 42, 501 (1981).

10. A. BAX, in "Topics in Carbon-13 NMR Spectroscopy" (G. C. Levy, Ed.), Vol. 4, Chap. 8, Wiley, New York, 1984.

11. H. Kessler, M. Bernd, H. Kogler, J. Zarbock, O. W. Sørensen, G. Bodenhausen, and R. R. ERnST, I. Am. Chem. Soc. 105, 6944 (1983).

12. H. Kessler, H. R. Loosl, ANd H. Oschinat, "Symposium Proceedings: Peptide" (U. Ragnarsson, Ed.), Almqvist \& Viksell, Stockholm, in press.

13. O. W. Sørensen, G. W. Eich, M. H. LeVitt, G. Bodenhausen, and R. R. ERnst, Progr. Nucl. Magn. Reson. Spectrosc. 16, 163 (1983).

14. K. J. PACKer AND K. M. Wright, Mol. Phys. 50, 797 (1983).

15. F. J. M. VAN De Ven and C. W. Hilbers, J. Magn. Reson. 54, 512 (1983).

16. A. BAX, J. Magn. Reson. 53, 149 (1983).

17. A. BaX AND G. Drobny, J. Magn. Reson. 61, 309 (1985).

18. G. Bodenhausen, R. Freeman, and D. L. Turner, J. Magn. Réson. 27, 511 (1977).

19. M. H. Levitt and R. Freeman, J. Magn. Reson. 33, 473 (1979).

20. M. H. LeVITT, J. Magn. Reson. 48, 234 (1982).

21. M. H. LevitT, J. Magn. Reson. 50, 95 (1982).

22. H. Kogler, O. W. Sørensen, G. Bodenhausen, AND R. R. ERnst, J. Magn. Reson. 55, 157 (1983).

23. A. BaX, R. A. Byrd, ANd A. Aszalos, J. Am. Chem. Soc. 106, 7632 (1984).

24. A. DEMARCO AND K. WÜthriCh, J. Magn. Reson. 24, 201 (1976).

25. U. Piantini, O. W. Sørensen, and R. R. ERnSt, J. Am. Chem. Soc. 104, 6800 (1982).

26. A. BaX, R. Freeman, AND G. A. Morris, J. Magn. Reson. 43, 333 (1981).

27. A. Bax, R. Freeman, and T. A. Frenkiel, J. Am. Chem. Soc. 103, 2102 (1981).

28. A. Bax, T. A. Frenkiel, R. Freeman, and M. H. Levitt, J. Magn. Reson. 43, 478 (1981).

29. T. H. Mareci and R. Freeman, J. Magn. Reson. 48, 158 (1982).

30. O. W. SøRENSEN AND R. R. ERNST, J. Magn. Reson. 55, 338 (1983). 